\title{
219. NUEVAS CITAS PARA LA FLORA VALLISOLETANA. V
}

Jesús Antonio LÁZARO BELLO

Recibido el 14 de octubre 2009, aceptado para su publicación el 5 febrero de 2011

New floristic records for Valladolid province. $V$

Palabras clave. Corología, flora vascular, España, Valladolid.

Key words. Chorology, vascular flora, Spain, Valladolid.

Continuando con una serie de trabajos anteriores (Lázaro Bello, 2002; 2003; 2005c; 2008b), se mencionan veintiseis taxones, de los cuales siete son novedades provinciales (señaladas con un *). Para los taxones, recogidos en diecinueve términos municipales diferentes, se siguen los criterios ya establecidos en los trabajos previos citados anteriormente. Los testimonios de los ejemplares citados se encuentran depositados en el herbario del Real Jardín Botánico de Madrid (MA).

Amaranthus blitoides S. Watson

VALLADOLID: Villafuerte, 30TUM8821, 760 m, 14-VII-2009, camino paralelo al río Esgueva, junto a una carretera, Lázaro Bello (MA 792214); Villanueva de los Caballeros, 30TUM1325, $710 \mathrm{~m}$, 19-VII-2009, taludes despejados y ruderalizados del río Sequillo, junto a una carretera, Lázaro Bello (MA 792207).

Especie altamente invasora, recientemente citada en la provincia de Valladolid (Lázaro Bello, 2005b; 2008b). La presente mención marca un nuevo límite noroccidental en su distribución peninsular (Sanz Elorza et al., 2004).

\section{Ammi majus L.}

*VALLADOLID: Simancas, 30TUM4805, 680 m, 20-VII-2009, terreno herboso en los alrededores de un parque, junto al río Pisuerga, Lázaro Bello (MA 792173); Zaratán, 30TUM5113, 760 m, 13-VI-2009, escombrera a las afueras del pueblo, Lázaro Bello (MA 792174).
Novedad provincial de una especie dispersa por buena parte de la Península Ibérica (Fuertes Aguilar, 2003).

\section{Barbarea vulgaris $\mathrm{R}$. Br.}

VALLADOLID: Villafuerte, 30TUM8821, 760 m, 9-VII-2009, camino paralelo al río Esgueva, Lázaro Bello (MA 792175); Villanueva de los Caballeros, 30TUM1325, 710 m, 19-VII-2009, orillas del río Sequillo, Lázaro Bello (MA 792176).

Conocemos una cita, de hace un siglo, del sur de la provincia (Gutiérrez Martín, 1908), y otra, más reciente, de la zona oriental (Romero Martín \& Rico, 1989).

\section{Beta maritima $\mathrm{L}$.}

*VALLADOLID: Medina de Rioseco, 30TUM3038, $720 \mathrm{~m}, 16-\mathrm{VII}-2009$, entorno ruderalizado del río Sequillo, Lázaro Bello (MA 792177); Villabrágima, 30TUM2433, $720 \mathrm{~m}$, 16-VII-2009, taludes ruderalizados del río Sequillo, Lázaro Bello (MA 792178); Villagarcía de Campos, 30TUM1728, $710 \mathrm{~m}, 17-\mathrm{VII}-2009$, entorno ruderalizado del río Sequillo, Lázaro Bello (MA 792179); Villanueva de los Caballeros, 30TUM1325, 710 m, 19-VII-2009, taludes del río Sequillo y aledaños, Lázaro Bello (MA 792180).

Primera cita provincial de un taxón que, a pesar de su adjetivo específico, se puede localizar en enclaves ruderalizados del interior peninsular (Gutiérrez Bustillo, 1990).

\section{Carex pseudocyperus L.}

VALLADOLID: Urueña, 30TUM1325, 710 
m, 19-VII-2009, orillas fangosas del río Sequillo, Lázaro Bello (MA 792181); Villagarcía de Campos, 30TUM1728, $710 \mathrm{~m}, 17-\mathrm{VII}-2009$, orillas del río Sequillo, Lázaro Bello (MA 792182).

La única alusión a su presencia en la provincia de Valladolid es la aportada por Luceño (2007) en Flora iberica.

\section{Chenopodium glaucum $\mathrm{L}$.}

*VALLADOLID: Tordesillas, 30TUL3296, $670 \mathrm{~m}, 2-\mathrm{VIII}-2009$, orillas algo fangosas del río Duero, en la zona de la playa, Lázaro Bello (MA 792186); Villanueva de Duero, 30TUL4699, 680 $\mathrm{m}, 14-\mathrm{IX}-2003$, arenas de donde se han retirado las aguas del río Adaja, Lázaro Bello (MA 792187).

Primera cita provincial de una especie que es rara en la región mediterránea, pero que se encuentra dispersa por la Península Ibérica (Uotila, 1990).

\section{Chenopodium urbicum $\mathrm{L}$.}

VALLADOLID: Villafuerte, 30TUM8821, 760 m, 14-VII-2009, medio ruderal situado entre una carretera, el río Esgueva y un cultivo de patata, sobre suelos algo margosos, Lázaro Bello (MA 792188).

Segunda cita provincial después de la de Gutiérrez Martín (1908), de Llano de Olmedo.

\section{Colutea brevialata Lange}

VALLADOLID: Cabezón de Pisuerga, 30TUM7119, 820 m, 24-V-2009, cuesta margosa, en ambiente de quejigar, Lázaro Bello (MA 792189); Tudela de Duero, 30TUM6704, 700 m, 3-VIII-2008, talud de caída al río Duero, en orla de pinar, Lázaro Bello (MA 792190).

Planta muy escasa en la provincia, citada en pocos enclaves y siempre con pequeño número de individuos (Fernández Alonso, 1986b; Gutiérrez Martín, 1908). Junto a las citas zamoranas de Bariego (2002), estas menciones son las más occidentales de la especie en la Península Ibérica (Talavera \& Arista, 1999).

Consolida ajacis (L.) Schur

VALLADOLID: Fuensaldaña, 30TUM5418, $760 \mathrm{~m}, 12-\mathrm{VI}-2009$, escombrera, sobre suelos arcillosos, junto a zona acarcavada, Lázaro Bello (MA 792191); Valbuena de Duero, 30TUM9409, $740 \mathrm{~m}, 7-\mathrm{VI}-2003$, talud de caída hacia el río Duero, Lázaro Bello (MA 792192).

Cultivada como ornamental, en ocasiones aparece naturalizada. Sólo conocemos referencias provinciales, sin aludir a términos municipales concretos, de Molero \& Blanché (1986), en Flora iberica, y de Sanz Elorza et al. (2008), en la reciente revisión que hacen de la flora alóctona localizada en Castilla y León.

Cucumis myriocarpus Naudin subsp. myriocarpus VALLADOLID: Puras, 30TUL6061, 790 $\mathrm{m}, 24-\mathrm{VII}-2009$, terreno inculto situado entre una escombrera y un campo de cultivo de girasol, Lázaro Bello (MA 792193).

Segunda cita provincial después de la de Burgaz \& Saiz (1989), de Almenara de Adaja, también en el sur de la provincia, aunque no recogida por Fernandes (1995) en Flora iberica.

\section{Cucurbita pepo L.}

*VALLADOLID: Bocigas, 30TUL5965, 770 m, 24-VII-2009, asilvestrada junto al paredón de una nave, a las afueras del pueblo, Lázaro Bello (MA 792194).

Planta hortícola que hemos encontrado asilvestrada sobre terreno ruderalizado y de la que no tenemos referencias para la provincia vallisoletana con esa condición.

\section{Datura innoxia Mill.}

VALLADOLID: Santovenia de Pisuerga, 30TUM5916, 700 m, 26-VII-2009, talud ruderalizado en las proximidades del río Pisuerga, Lázaro Bello (MA 792195).

Encontrada en los últimos años en la provincia de Valladolid (Sanz Elorza et al., 2006; Sanz Elorza \& González Bernardo, 2007), aportamos una nueva localidad de la cuenca del Duero, en donde es muy escasa, como se pone de manifiesto en el mapa de distribución mostrado en Sanz Elorza et al. (2004).

\section{Gnaphalium luteo-album $\mathrm{L}$.}

VALLADOLID: Valoria la Buena, 30TUM7231, 720 m, 13-VIII-2004, depresiones temporalmente húmedas, salinas y muy ruderalizadas, Lázaro Bello (MA 792196); ibidem, 27-VI-2009, Lázaro Bello (MA 792197)

Especie previamente recogida por Gutiérrez Martín (1908) en el sur de la provincia.

\section{Knautia subscaposa var. subintegerrima Rouy} VALLADOLID: Castrodeza, 30TUM3712, 
820 m, 17-V-2009, cuesta margosa, Lázaro Bello (MA 792198).

Aunque se conocen dos citas previas de la especie en la provincia vallisoletana (Fernández Alonso, 1986a; Lázaro Bello, 2008a), la variedad aquí mencionada, especialmente abundante en el sureste de España, sólo aparece recogida en Flora iberica (Devesa, 2007). Está caracterizada por tener todas o la mayoría de las hojas enteras, o, a veces, alguna laxamente dentada, pinnatífida o lobulada,

Lappula squarrosa (Retz.) Dumort.

VALLADOLID: Cabezón de Pisuerga, 30TUM6722, $860 \mathrm{~m}, 27-\mathrm{VI}-2009$, talud margoso, en el entorno de una escombrera, Lázaro Bello (MA 792199).

Hasta ahora, sólo una cita antigua, de Willkomm \& Lange (1870), alude a su presencia en la provincia de Valladolid.

\section{Lepidium graminifolium $\mathrm{L}$.}

VALLADOLID: Villanueva de los Caballeros, 30TUM1325, $710 \mathrm{~m}, 19-\mathrm{VII}-2009$, taludes despejados y ruderalizados del río Sequillo, Lázaro Bello (MA 792200).

Taxón más frecuente en la mitad este de la Península Ibérica (Hernández Bermejo \& Clemente, 1993), del que se conocen dos citas vallisoletanas bastante próximas entre sí, en la zona oriental de la provincia (Fernández Alonso, 1986a; Romero Martín \& Rico, 1989).

\section{Orchis langei $\mathrm{K}$. Richt.}

*VALLADOLID: Cabezón de Pisuerga, 30TUM7119, 820 m, 24-V-2009, cuesta margosa, en ambiente de quejigar, Lázaro Bello (MA 792201).

Novedad provincial de una especie dispersa por casi toda la Península Ibérica (Aedo, 2005), y que hay que añadir al reciente catálogo de orquídeas conocidas en la provincia de Valladolid aportado por Santos Vicente et al. (2008).

Pisum sativum subsp. sativum var. arvense (L.) Poir.

*VALLADOLID: Robladillo, 30TUM4108, $840 \mathrm{~m}, 30-\mathrm{V}-2009$, asilvestrado en bordes de caminos y de cultivos, Lázaro Bello (MA 792202).

Primera cita provincial de un taxón que es frecuente encontrar cultivado en su variedad sativum, pero del que aportamos pliego testigo de la variedad arvense, que aparece asilvestrada de forma esporádica.

Populus $x$ canescens (Aiton) Sm.

VALLADOLID: Tordesillas, 30TUL3296, 670 m, 2-VIII-2009, chopera junto a la vegetación de ribera del río Duero, Lázaro Bello (MA 792203).

Aunque Soriano (1995), para Flora iberica, no recoge el taxón en la provincia vallisoletana, Oria de Rueda \& Diez (2003), en un texto de carácter más bien divulgativo, sí lo recogen en un mapa de distribución de la especie en la comunidad castellano-leonesa.

\section{Prangos trifida (Mill.) Herrnst. \& Heyn}

VALLADOLID: Cabezón de Pisuerga, 30TUM7120, $860 \mathrm{~m}, 24-\mathrm{V}-2009$, claros de encinar en suelos pedregosos de paramera calcárea, Lázaro Bello (MA 792204).

Planta muy escasa en la provincia vallisoletana que siempre se ha mencionado como localizada en el término municipal de Renedo de Esgueva (Fernández Díez, 1984; Lázaro Bello, 2002; 2004; 2005a; 2006), pero de la que hemos encontrado otra población, en una cuadrícula UTM de 10 x $10 \mathrm{~km}$ diferente, situada más al norte.

Ranunculus bulbosus subsp. aleae (Willk.) Rouy \& Foucaud

VALLADOLID: Wamba, 30TUM4015, 780 $\mathrm{m}, 10-\mathrm{V}-2009$, pastizal en el entorno del arroyo de Hontanija, Lázaro Bello (MA 792205).

Segunda cita provincial, tras la dada hace un siglo, por Gutierrez Martín (1908), para el partido judicial de Olmedo.

\section{Rorippa amphibia (L.) Besser}

VALLADOLID: Simancas, 30TUM4805, 680 m, 7-VI-2009, orillas del río Pisuerga, junto a una cascajera, Lázaro Bello (MA 792206); Valoria la Buena, 30TUM6929, 700 m, 23-V-2009, orillas del río Pisuerga, Lázaro Bello (MA 792208).

Especie sólo citada por Sánchez Sánchez \& Fernández Díez (1986), para el municipio de Castronuño.

Sinapis alba subsp. mairei (H. Lindb. fil.) Maire

VALLADOLID: Simancas, 30TUM4805, 680 m, 7-VI-2009, cascajera del río Pisuerga, Lázaro Bello (MA 792209).

Taxón recientemente señalado por Lázaro Bello 
(2005c) en el municipio de Santovenia de Pisuerga, que parece escasa a nivel provincial.

\section{Thalictrum tuberosum $\mathrm{L}$.}

VALLADOLID: Cabezón de Pisuerga, 30TUM7021, 860 m, 24-V-2009, paramera calcárea, en ambiente de encinar, Lázaro Bello (MA 792210); Castrillo de Duero, 30TVM1506, 820 m, 29-V-2004, quejigar en cuesta margo-calcárea, Lázaro Bello (MA 792211).

Únicamente conocemos la cita de Fernández Díez (1984), aludiendo a su presencia en el valle del Esgueva.

\section{Vicia tenuifolia Roth}

VALLADOLID: Cabezón de Pisuerga, 30TUM7119, 820 m, 24-V-2009, cuesta margosa, en ambiente de quejigar, Lázaro Bello (MA 792212).

Segunda cita provincial, después de la de Gutiérrez Martín (1908), que ya la señaló, hace un siglo, en el partido judicial de Olmedo, en el sur de la provincia.

\section{Yucca aloifolia $\mathrm{L}$}

*VALLADOLID: Simancas, 30TUM4807, 720 m, 7-VI-2009, asilvestrada en terreno inculto, junto a una carretera, Lázaro Bello (MA 792213).

Planta alóctona de origen neotropical de la que conocemos una alusión, como ornamental, en la ciudad de Valladolid (López Gómez \& Díez, 1991), pero que hemos encontrado asilvestrada en la zona centro de la provincia.

\section{BIBLIOGRAFÍA}

AEDO, C. -2005- Orchis L. In: C. Aedo \& A. Herrero (eds.). Flora iberica XXI: 114-146.

BARIEGO HERNÁNDEZ, P. -2002- Sobre la presencia de Colutea brevialata Lange en la provincia de Zamora. Stud. Bot, Univ. Salamanca 20: $149-150$

BURGAZ, A.R. y F. SAIZ ALCÁNTARA -1989Estudio fenológico de las comunidades de Tierra de Pinares (Valladolid, España). Bot. Complutensis 15: 127-147.

DEVESA, J.A. -2007- Knautia L. In: J.A. Devesa et al. (eds.). Flora iberica XV: 286-305.

FERNANDES, R.B. -1995- Cucumis L. In: S. Castroviejo et al. (eds.). Flora iberica III
461-466

FERNÁNDEZ ALONSO, J.L. -1986a- Fragmenta chorologica occidentalia, 306-392. Anales Jard. Bot. Madrid 42(2): 517-525.

FERNÁNDEZ ALONSO, J.L. -1986b- Fragmenta chorologica occidentalia, 494-521. Anales Jard. Bot. Madrid 43(1): 161-164.

FERNÁNDEZ DÍEZ, F.J. -1984- Aportaciones a la flora vallisoletana. Stud. Bot., Univ. Salamanca 3: 255-262.

FUERTES AGUILAR, J. -2003- Ammi L. In: G. Nieto Feliner et al. (eds.). Flora iberica X: 288-290.

GUTIÉRREZ BUSTILLO, A.M. -1990- Beta L. In: S. Castroviejo et al. (eds.). Flora iberica II: 478-482.

GUTIÉRREZ MARTÍN, D. -1908-Apuntes para la flora del Partido Judicial de Olmedo e indicaciones de los usos medicinales que algunas plantas reciben. Tip. Benito Manuel. Ávila.

HERNÁNDEZ BERMEJO, J.E. y M. CLEMENTE -1993- Lepidium (L.) R. Br. In: S. Castroviejo et al. (eds.). Flora iberica IV: 311-327.

LÁZARO BELLO, J.A. -2002- Nuevas citas para la flora vallisoletana. Acta Bot. Malacitana 27: 249-253.

LÁZARO BELLO, J.A. -2003- Nuevas citas para la flora vallisoletana. II. Acta Bot. Malacitana 28: 184-188.

LÁZARO BELLO, J.A. -2004- La diversidad florística en Renedo de Esgueva (Valladolid). In: R. Hernández Muñoz et al. (eds.). Libro de Investigación 2: 421-437. I.E.S. Félix Rodríguez de la Fuente. Burgos.

LÁZARO BELLO, J.A. -2005a- Estudio de diversidad florística en páramos de la zona centro de la cuenca del Duero (Valladolid, España). Toll Negre 6: 25-34.

LÁZARO BELLO, J.A. -2005b- Notas corológicas para la flora vascular de la zona centro de la cuenca del Duero (Valladolid, España). Anales de Biología, Univ. Murcia 27: 113-118.

LÁZARO BELLO, J.A. -2005c- Nuevas citas para la flora vallisoletana. III. Acta Bot. Malacitana 30: 177-181.

LÁZARO BELLO, J.A. -2006- Renedo de Esgueva (Valladolid, España): Catálogo florístico y análisis de resultados. Ecología 20: 163-216.

LÁZARO BELLO, J.A. -2008a- Novedades florísticas en los Montes de Torozos (Valladolid, 
España). Flora Montiberica 38: 9-12.

LÁZARO BELLO, J.A. -2008b- Nuevas citas para la flora vallisoletana. IV. Acta Bot. Malacitana 33: 334-338.

LÓPEZ GÓMEZ, M.T. y R. DOMINGO DÍEZ -1991- Campo Grande, jardín histórico de Valladolid. Junta de Castilla y León. Valladolid. LUCEÑO, M. -2007- Carex L. In: S. Castroviejo et al. (eds.). Flora iberica XVIII: 109-250.

MOLERO, J. \& C. BLANCHÉ. -1986- Consolida (DC.) S.F. Gray. In: S. Castroviejo et al. (eds.). Flora iberica I: 252-255.

ORIA DE RUEDA, J.A. y J. DIEZ -2003-Guía de árboles y arbustos de Castilla y León. Ediciones Cálamo. Palencia.

ROMERO MARTÍN, T. y E. RICO -1989- Flora de la cuenca del río Duratón. Ruizia 8: 1-438.

SÁNCHEZ SÁNCHEZ, J. \& F.J. FERNÁNDEZ DÍEZ -1986- Notas sobre flora vallisoletana. Lazaroa 9: 181-187.

SANTOS VICENTE, M., P. BARIEGO HERNÁNDEZ, L. DELGADO SÁNCHEZ, L.P. GAVILÁN IGLESIAS y A. GASTÓN GONZÁLEZ -2008- Aproximación al catálogo de las orquídeas de la provincia de Valladolid. Bot. Complutensis 32: 139-147.

SANZ ELORZA, M., E.D. DANA SÁNCHEZ y E. SOBRINO -2004- Atlas de las Plantas Alóctonas invasoras en España. Ministerio de Medio Ambiente. Madrid.

SANZ ELORZA, M., E.D. DANA SÁNCHEZ y E. SOBRINO -2006- Aportaciones a la flora de la provincia de Segovia (España) III. Bot. Complutensis 30: 105-111.

SANZ ELORZA, M. y F. GONZÁLEZ BERNARDO -2007- Contribución al conocimiento de la flora vascular alóctona de Castilla y León. Stud Bot., Univ. Salamanca 26: 105-110.

SANZ ELORZA, M., F. GONZÁLEZ BERNARDO y L.P. GAVILÁN IGLESIAS -2008- La flora alóctona de Castilla y León (España). Bot. Complutensis 32: 117-137.

SORIANO, C. -1995- Populus L. In: S. Castroviejo et al. (eds.). Flora iberica III: 471-477.
TALAVERA, S. y M. ARISTA. -1999- Colutea L. In: C. Aedo et al. (eds.). Flora iberica VII(I): 274-279.

UOTILA, P. -1990- Chenopodium L. In: S. Castroviejo et al. (eds.). Flora iberica II: 484-500.

WILLKOMM, M. \& J. LANGE -1870- Boraginaceae Juss. In: Willkomm \& Lange (eds.). Prodromus Florae Hispanicae II: 482-513. Stuttgart.

Dirección del autor. C/ Madre de Dios $\mathrm{n}^{\circ} 15,1^{\circ} \mathrm{D}$ 47011-Valladolid (España). E-mail: chuchijalb@ hotmail.com 